\title{
AGN Feedback in Galaxy Groups: A Detailed Study of X-Ray Features and Diffuse Radio Emission in IC 1262
}

\author{
M. B. Pandge ${ }^{1,9}$ (), S. S. Sonkamble ${ }^{2}$ (1), Viral Parekh ${ }^{3}$, Pratik Dabhade ${ }^{4,5}$, Avni Parmar $^{3}$, \\ M. K. Patil ${ }^{6}$, and Somak Raychaudhury ${ }^{4,7,8}$ (i) \\ ${ }^{1}$ SERB Young Scientist, Dayanand Science College, Barshi Road Latur, 413 512, India; mbpandge@associates.iucaa.in, mbpandge@gmail.com \\ ${ }^{2}$ National Center for Radio Astrophysics (NCRA), Tata Institute of Fundamental Research (TIFR), Pune 411 007, India \\ ${ }^{3}$ Raman Research Institute, C.V. Raman Avenue, Sadashivnagar, Bangalore 560080, India \\ ${ }^{4}$ Inter-University center for Astronomy and Astrophysics, Post Bag 4, Ganeshkhind, Pune-411 007, India \\ ${ }^{5}$ Leiden Observatory, Leiden University, Niels Bohrweg 2, 2333 CA, Leiden, The Netherlands \\ ${ }^{6}$ School of Physical Sciences, Swami Ramanand Teerth Marathwada University Nanded, 431606 , India \\ ${ }^{7}$ Department of Physics, Presidency University, 86/1 College Street, Kolkata 700073, India \\ ${ }^{8}$ School of Physics and Astronomy, University of Birmingham, Birmingham B15 2TT, UK \\ Received 2018 January 19; revised 2018 October 31; accepted 2018 November 12; published 2019 January 8
}

\begin{abstract}
This paper reports a systematic search of X-ray cavities, density jumps, and shocks in the intergalactic environment of the galaxy group IC 1262 using Chandra, GMRT, and Very Large Array archival observations. The X-ray imaging analysis reveals a pair of X-ray cavities to the north and south of the X-ray peak, at projected distances of $6.48 \mathrm{kpc}$ and $6.30 \mathrm{kpc}$ respectively. Total mechanical power contained in both of these cavities is found to be $\sim 12.37 \times 10^{42} \mathrm{erg} \mathrm{s}{ }^{-1}$, and compares well with the X-ray luminosity, within the cooling radius, measured to be $\sim 3.29 \times 10^{42}$ erg s ${ }^{-1}$, suggesting that the mechanical power injected by the central AGN efficiently balances the radiative loss. We detect a previously unknown X-ray cavity at the position of the southern radio lobe in the intra-group medium and find a loop of excess X-ray emission extending $\sim 100 \mathrm{kpc}$ southwest from the central galaxy. The X-ray cavity at the position of the southern radio lobe probably represents a first generation X-ray cavity. Two surface brightness edges are evident to the west and northeast of the center of this group. The radio galaxy at the core of the IC 1262 group is a rare low-redshift ultra-steep radio galaxy, its spectral index being $\alpha \sim-1.73$ (including the central AGN) and $\alpha \sim-2.08$ (excluding the central AGN). We detect a radio phoenix embedded within the southern radio lobe, for the first time in a poor group, with a spectral index $(\alpha \leqslant-1.92)$. The spectral index distribution across the phoenix steepens with increasing distance from its intensity peak.
\end{abstract}

Key words: galaxies: active - galaxies: general - galaxies: groups: individual (IC 1262)

\section{Introduction}

High resolution X-ray images from the new generation X-ray telescopes, Chandra and XMM-Newton, have provided us with mounting evidence of various modes of interaction between the hot intergalactic gas, and the active galactic nucleus at the core of the clusters (e.g., Perseus), groups (e.g., NGC 5044), and ellipticals (e.g., Cygnus A). This interaction may also result in the formation of the substructures or cavities apparent in the surface brightness distribution of the X-ray emission (Rafferty et al. 2006; Bîrzan et al. 2008; David et al. 2009; Dunn et al. 2010; O'Sullivan et al. 2011; Bîrzan et al. 2012; Chon et al. 2012). Radio observations indicate that many of these cavities are associated with enhanced level synchrotron emitting cosmic-ray particles of either charge. As the cosmic rays provide enough pressure without appreciable increase in the mass density, these cavities or bubbles are found to rise buoyantly in the hot gaseous environment. These cavities can be as small as $\sim 5 \mathrm{kpc}$ in diameter, and as large as $\sim 200 \mathrm{kpc}$ located in the central $\sim 20 \mathrm{kpc}$ of a cluster, or in its outskirts. They are seen in giant elliptical galaxies (Jones et al. 2002) and groups (Machacek et al. 2006; Jetha et al. 2008; Giacintucci et al. 2011, 2012), as well as in galaxy clusters (McNamara et al. 2000; Bîrzan et al. 2004; Dunn \& Fabian 2006; Rafferty et al. 2006). The mechanical power required for "inflating" these cavities matches well with the radiative cooling loss in many of the cluster cores, suggesting that

\footnotetext{
${ }^{9}$ DST INSPIRE Faculty.
}

these bubbles, and perhaps the cosmic rays that fill them, are involved in the feedback process.

Most studies of AGN feedback seem to concern massive clusters (e.g., the analysis of cluster samples by Dunn \& Fabian 2006; Bîrzan et al. 2008; Mittal et al. 2009), yet most of the galaxies in the universe are found in smaller systems, such as poor clusters and groups (Eke et al. 2004). Complete volumelimited studies of galaxy groups are rare (e.g., O'Sullivan et al. 2017), but these reveal the diversity of physical phenomena that exist in the intergalactic medium of groups due to close interactions in the sluggish environment of groups. A systematic study of nearby galaxy groups with AGN-ICM interactions is important to understand the AGN feedback in relatively smaller dark matter halos. Due to their shallower gravitational potentials, the AGN outbursts in such systems are believed to produce a larger impact on the intra-group medium (IGM).

This study is also important because the relationship between the AGNs and intergalactic hot gas (e.g., Giacintucci et al. 2011) and the atomic and molecular gas (e.g., O'Sullivan et al. 2018) can significantly influence galaxy evolution in the group environment. Nearby groups are also useful to probe regions closer to the central black hole, in the brightest galaxy in the core, in greater detail, due to proximity and better signal. We have thus chosen to perform a systematic study of the impact of AGN feedback on the IGM in the nearby galaxy group IC 1262, which has the eponymous dominant early-type galaxy, and 31 members within $\left(20^{\prime} \times 20^{\prime}\right)^{2}$ (Smith et al. 2004). 
Trinchieri \& Pietsch (2000) reported a bright arc in close proximity of the $\mathrm{cD}$ galaxy, which may have resulted from the dynamic evolution of the central galaxy due to a merger event in this poor group. Hudson \& Henriksen (2003) and Hudson et al. (2003) studied this group using BeppoSAX and Chandra in the X-ray and the NRAO Very Large Array (VLA) Sky Survey and the Westerbork Northern Sky Survey measurements in the radio, to claim the detection of diffuse nonthermal emission to the South of IC 1262, likely associated with a radio mini-halo, produced by an earlier merger event. These authors also detected a diffuse radio structure of spectral index $\sim 1.8$, slightly more extended than the central cD galaxy. Furthermore, Trinchieri et al. (2007) identified and confirmed certain signatures of merging, such as surface brightness discontinuities, with disturbed structures in the core and the sharp and narrow filamentary structures east of the central galaxy, using high resolution X-ray images from Chandra and $X M M-N e w t o n$. The above studies have in general concluded that high resolution, deeper radio observations are needed for IC 1262 in order to understand the radio properties in more detail. This group is located at a relatively low redshift of $z=0.0326$, and its central galaxy hosts the radio source $4 \mathrm{C}+43.46$, whose radio power is given by $\log P_{1.4 \mathrm{GHz}} \leqslant 22.56 \mathrm{~W} \mathrm{~Hz}^{-1}$ (Trinchieri et al. 2007; Dong et al. 2010). A single X-ray cavity was detected by Dong et al. (2010) toward the north of the center of the group. It is interesting to note that the central galaxy IC 1262 shows very little star formation $\left(\sim 4.35 \times 10^{-2} M_{\odot} \mathrm{yr}^{-1}\right)$ (Vaddi et al. 2016). In this work, we have tried to assemble available multiwavelength data from different archives such as Chandra, SDSS, GMRT, and VLA in order to understand the nature of this group, in particular, to understand the relation between its merger history and the various X-ray and radio features that are detected, and to understand the AGN feedback process operating in this group. In addition, we use high resolution radio images to understand the origin of more complex radio structure detected in the southern radio lobe (Hudson et al. 2003). The structure of the paper is as follows: In Section 2 we present the data analysis, while the detection of the X-ray cavity is outlined discussed in Section 3. Section 4 describes the X-ray spectral analysis. Finally, we present the results from the radio analysis in Section 5. Throughout this paper we assume $\Lambda$ CDM cosmology with $H_{0}=70 \mathrm{~km} \mathrm{~s}^{-1} \mathrm{Mpc}^{-1}, \Omega_{M}=0.27 \& \Omega_{\Lambda}=0.73$, translating to a scale of $0.639 \mathrm{kpc} \operatorname{arcsec}^{-1}$ at the redshift $z=0.032$ of IC 1262 . All spectral analysis errors are at $90 \%$ confidence, while all other errors are at $68 \%$ confidence.

\section{Data Analysis}

\subsection{X-Ray Observations}

The field of IC 1262 was observed three times in X-rays by the Chandra X-ray Observatory between 2006 April 17-22, for an effective exposure of $120 \mathrm{ks}$ (ObsID and other details in Table 1). The observations were reprocessed using the CHANDRA_REPRO task available within CIAO ${ }^{10} 4.8$ and employing the latest calibration files CALDB 4.7.2 provided by the Chandra X-ray center (CXC). We followed the standard Chandra data-reduction threads ${ }^{11}$ for the analysis. Periods of high background flares were identified using the $l c \_s i g m a \_c l i p$ algorithm, with the threshold set at $3 \sigma$. These periods were removed from further analysis. The CIAO REPROJECT_OBS

\footnotetext{
${ }^{10}$ http://cxc.harvard.edu/ciao

11 http://cxc.harvard.edu/ciao/threads/index.html
}

Table 1

Chandra Observation Log

\begin{tabular}{lccccc}
\hline \hline ObsID & $\begin{array}{c}\text { Observing } \\
\text { Mode }\end{array}$ & CCDs on & Starting Date & $\begin{array}{c}\text { Total } \\
\text { Time } \\
(\mathrm{ks})\end{array}$ & $\begin{array}{c}\text { Clean } \\
\text { Time } \\
(\mathrm{ks})\end{array}$ \\
\hline 6949 & VFAINT & $0,1,2,3,6$ & 2006 Apr 17 & 40 & 37.54 \\
7321 & VFAINT & $0,1,2,3,6$ & 2006 Apr 19 & 40 & 35.97 \\
7322 & VFAINT & $0,1,2,3,6$ & 2006 Apr 22 & 40 & 37.47 \\
\hline
\end{tabular}

script was used to reproject the event files and exposure maps in the energy band $0.7-7.0 \mathrm{keV}$ were extracted using the FLUX_OBS script. CIAO scripts blanksky and blanksky_image were used to identify suitable blank sky background fields, corresponding to each of the event files, and were used for removing the particle background contamination. Point sources were identified using the WAVDETECT algorithm within CIAO and were removed from the image. Spectra and corresponding Redistribution Matrix Files (RMF), Ancillary Response Files (ARF) were generated using the SPECEXTRACT task within CIAO 4.8.

\subsection{Radio Observations}

Archival high frequency VLA and low frequency GMRT data for IC 1262 used, whose observational details are summarized in Table 2.

\subsubsection{VLA}

We used VLA archival radio data for the field IC 1262, using $L$-band continuum $(1400 \mathrm{MHz})$ observations in the B (max baseline $11.1 \mathrm{~km})$ and $\mathrm{D}(\max$ baseline $1.03 \mathrm{~km})$ configurations. These observations were analyzed following the standard routine and using the Common Astronomy Software Applications (CASA) package of version 4.6.0. The data were inspected for RFI (radio frequency interference), non-working antennas, bad baselines, channels, and time period. Corrupted data were excised from the $u-v$ data set. The flux density of each primary or flux calibrator was set according to Perley \& Butler (2017). The list of both primary and secondary calibrators is given in Table 2. The same flux calibrator was used for the bandpass calibration followed by determining the flux density of the secondary or phase calibrator(s) using the antenna complex gain solutions. Calibrated visibilities were then used to create the images by the standard Fourier transform deconvolution. A few rounds of self-calibration ( 2 phase +1 amplitude) were applied to reduce the effects of residual phase errors in the data and to improve the quality of the final images. We produced images with the robust " 0 " parameter in the "clean" task of CASA.

\subsubsection{GMRT}

GMRT archival (Project code 07MHA01) $P$-band data of $16 \mathrm{MHz}$ bandwidth centered at $325 \mathrm{MHz}$ frequency and TGSS $150 \mathrm{MHz}$ (TIFR-GMRT SKY SURVEY, ${ }^{12}$ data; Intema et al. 2017) was used for low frequency study of IC 1262. The $325 \mathrm{MHz} P$-band data was processed using Source Peeling and Atmospheric Modeling (SPAM; Intema 2014), an AIPS-based semi-automated pipeline radio data-reduction package that performs flagging, initial-calibration and imaging with

\footnotetext{
$\overline{12 \text { http://tgssadr.strw.leidenuniv.nl/doku.php }}$
} 

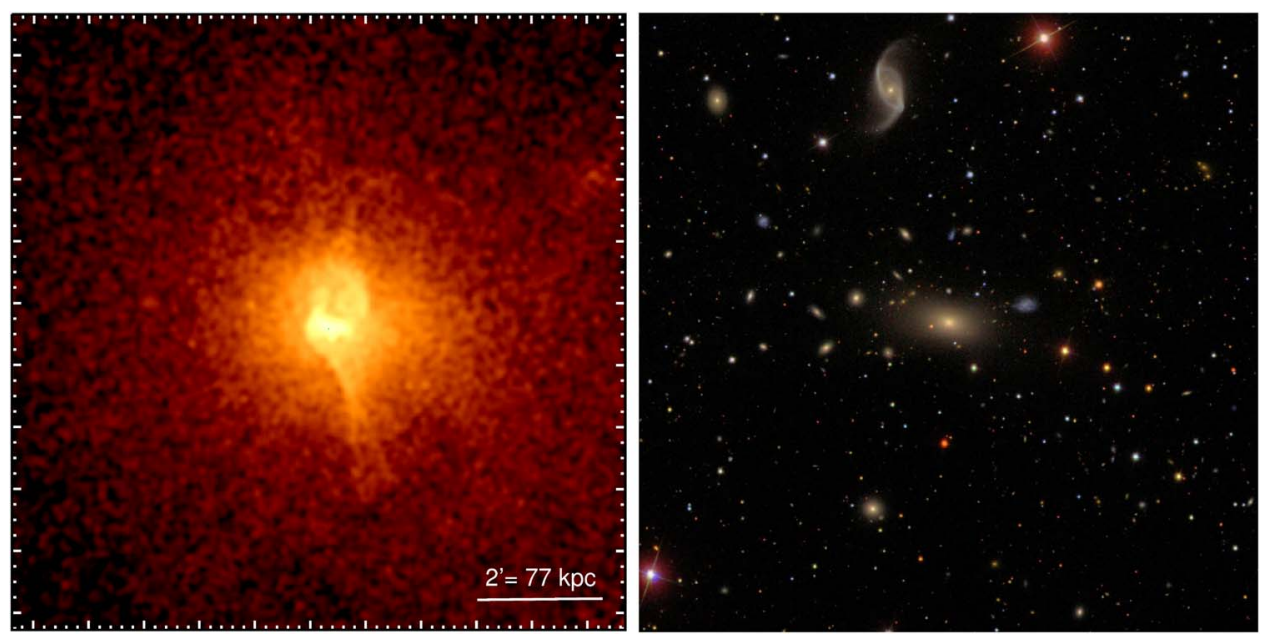

Figure 1. Left panel: the $0.7-2.0 \mathrm{keV}$ background-subtracted, exposure-corrected $10^{\prime} \times 10^{\prime}$ Chandra X-ray image of the IC 1262 group. The X-ray image is smoothed by a $3 \sigma$ Gaussian ( $\sigma=1$ pixel). Right panel: optical counterpart of IC 1262 in the $R$-band, from the Sloan Digital Sky Survey.

Table 2

IC 1262 Radio Data

\begin{tabular}{|c|c|c|c|c|c|}
\hline Telescope & Project Code & Frequency (MHz) & Telescope Configuration & Date of obs & Total obs Time (minutes) \\
\hline VLA & S7601 & 1400 & B & 2006 Jul 27 & 38 \\
\hline VLA & S7601 & 1400 & B & $2006 \mathrm{Jul} 28$ & 18 \\
\hline VLA & S7601 & 1400 & $\mathrm{D}$ & 2007 May 13 & 120 \\
\hline GMRT & 07МНА01 & 325 & $\cdots$ & 2005 Mar 4 & 400 \\
\hline GMRT & 16_279 & 150 & $\cdots$ & 2011 Jun 17 & 10 \\
\hline
\end{tabular}

direction dependent calibration in an iterative way. The complete working of SPAM can be seen in Intema et al. (2017). The same package was used for processing the entire TGSS data by Intema et al. (2017).

\section{X-Ray Imaging Analysis}

The background-subtracted, exposure-corrected, adaptively smoothed $0.7-2.0 \mathrm{keV}$ Chandra image of this group is shown in Figure 1 (left panel). For comparison, we also show its optical counterpart $R$-band image taken from the Sloan Digital Sky Survey (right panel, $\operatorname{SDSS}^{13}$ ) on the same scale, which confirms the extended nature of the X-ray emission from this system. This figure reveals interesting structures in the X-ray image such as surface brightness edges, and a narrow X-ray ridge in the central region of this group. These structures are consistent with those reported by Trinchieri et al. (2007).

To understand the broad spectral properties of the X-ray map, we generated a tricolor image in the energy range $0.7-8.0 \mathrm{keV}$, where, from the Chandra observation, X-ray photons were extracted in three different energy bands, namely, soft $(0.7-1.0 \mathrm{keV})$, medium $(1-2.0 \mathrm{keV})$ and hard $(2-8.0 \mathrm{keV})$. These were smoothed, setting the parameters sigmin $=3$ and sigmax $=5$, and were combined to generate the tricolor image for this system (Figure 2). The red color in these image represents the soft component of the X-ray emitting gas, while the medium and hard components are represented in green and blue respectively. This broadband spectral image highlights the presence of several substructures in this system.

\footnotetext{
${ }^{13}$ http://www.sdss.org/
}

\subsection{Detection of X-Ray Cavities}

A visual inspection of the $0.7-2 \mathrm{keV}$ Chandra image (Figure 1) of IC 1262 reveals structures like depressions or cavities in the surface brightness distribution of the hot ICM, of the kind reported in Dong et al. (2010), where the existence of cavities were discussed, but their position and size were not measured. Here we identify the cavities and quantify them using two different techniques.

\subsubsection{Unsharp Masked Image}

The 0.7-2 keV Chandra image of the IC 1262 group, after exposure correction and subtraction of background, was subjected to a procedure of unsharp masking, a procedure often used to reveal underlying structures. The image was first smoothed with a $2 \sigma$ wide Gaussian kernel, using the task aconvolve within CIAO. This suppresses the pixel-to-pixel variations of the X-ray brightness, while preserving small-scale uncorrelated structures. A similar image was also generated by smoothing it with a $10 \sigma$ wider Gaussian kernel, which erases small-scale features, while preserving the overall morphology of the hot gas distribution in this group. The unsharp masked image was then generated by subtracting the image smoothed with $10 \sigma$ from that smoothed with a $2 \sigma$ wide Gaussian kernel. The resulting unsharp masked image is shown in Figure 3 (left panel). A careful inspection of this figure confirms the presence of X-ray depressions or cavities in the surface brightness distribution of the hot gas from IC 1262. In addition to the obvious depressions or cavities, there are some regions delineating excess emission, as compared to the average surface brightness. 


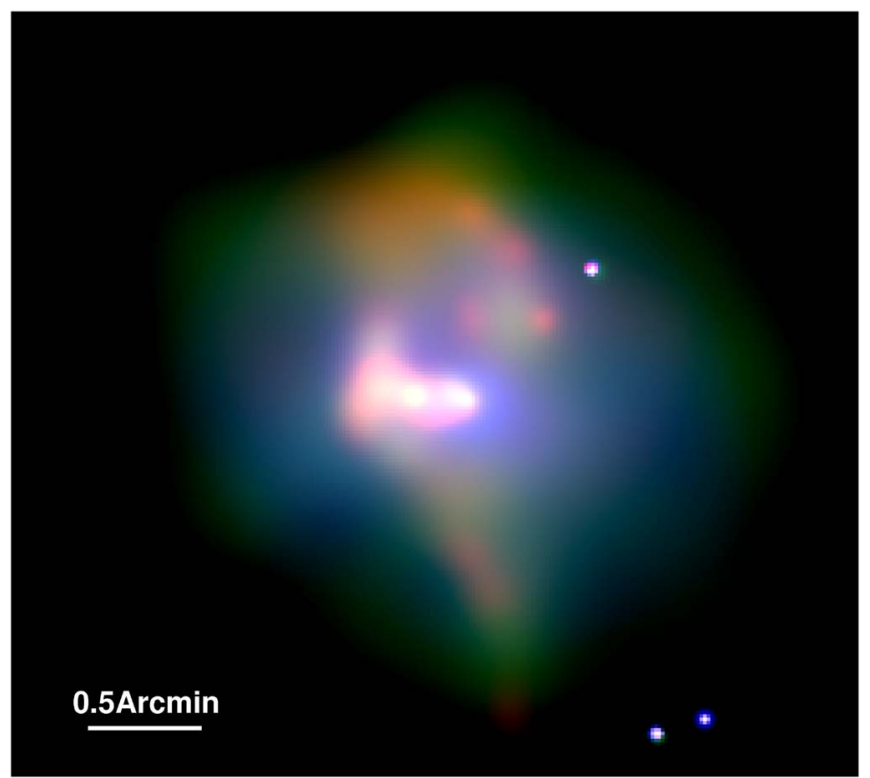

Figure 2. Tricolor Chandra X-ray image of IC 1262. The soft X-ray image $(0.7-1 \mathrm{keV})$ is shown in red, the image in the intermediate $(1-2 \mathrm{keV})$ band is shown in green, and that in the hard $(2-8 \mathrm{keV})$ band is shown in blue.

\subsection{2. $\beta$-model Subtracted Residual Maps}

Further confirmation of the X-ray cavities and excess emission evident in the unsharp masked image, a $2 \mathrm{D} \beta$-model-subtracted residual image of IC 1262 was generated. For this, we first generated the two-dimensional smooth model by fitting ellipses to the isophotes in the clean background-subtracted, pointsource-removed X-ray image, using the fitting package Sherpa available within CIAO. The model parameters i.e., ellipticity, position angle, normalization angle, and local background, etc., were kept free during the fit.

The best-fit 2D model was then subtracted from the background-subtracted, exposure-corrected Chandra image of IC 1262 to produce its residual map, which is shown in Figure 3 (right panel). This confirms all the major features evident in the unsharp masked image. The $1.4 \mathrm{GHz}$ (green) contours (from the VLA observation) are overlaid on the unsharp-masked image. The contours are at levels $2.5 \sigma, 3.0 \sigma$, and $3.5 \sigma$, where $\sigma$ is $6 \mu \mathrm{Jy}_{\text {beam }^{-1}}$ for the VLA-B configuration.

Both these images confirm the presence of a loop-like structure or an arc (see Figure 3 left panel) directed $\sim 38^{\prime \prime}$ northwest of IC 1262. In comparable groups or clusters (e.g., Gitti et al. 2010; David et al. 2011), dark cavities are normally seen in pairs on either side of the central excess emission, and are believed to be the result of the interaction of radio jets from a central dominant system with the surrounding IGM, in the form of buoyantly rising bubbles. In this system, we detect two X-ray cavities toward the north (hereafter Ncavity) and south (hereafter Scavity) from the center: these are highlighted by white arrows in both the images. The detected X-ray cavities and their morphological parameters are tabulated in Table 3 . In this table, columns 3 and 4 show the semimajor and semiminor axes of the cavities, while column 5 gives their projected distance from the center of the group.

\subsection{Surface Brightness Edges}

In Figures 1-3, we find clear hints of surface brightness edges (hereafter SBEs) along the east and northwest directions, at $\sim 38^{\prime \prime}$ and $45^{\prime \prime}$ respectively, from the center of IC 1262. In order to investigate the origin of these SBEs, we extract surface brightness profiles of the X-ray emission in the energy range 0.7-4.0 keV, using PROFFIT-V1.4 (Eckert et al. 2011), in the annular regions $(\mathrm{A})\left(30^{\circ}-110^{\circ}\right)$ and $(\mathrm{B})\left(120^{\circ}-270^{\circ}\right) 5$ (shown in yellow in Figure 3 ). The extracted profiles along the east and northwest edges are shown in Figure 4. These figures indicate that the apparent sharp changes in the surface brightness are due the existence of edges along the respective directions.

The extracted surface brightness profiles across these edges A and B were fitted with a broken power-law density model. In both cases, clear density compressions, at levels of over $90 \%$ confidence, are evident. The broken power-law density model is parameterized as

$$
n(r)=\left\{\begin{array}{ll}
\mathcal{C} n_{0}\left(\frac{r}{r_{\mathrm{sh}}}\right)^{-\alpha 1}, & \text { if } r<r_{\mathrm{sh}} \\
n_{0}\left(\frac{r}{r_{\mathrm{sh}}}\right)^{-\alpha 2}, & \text { if } r>r_{\mathrm{sh}}
\end{array},\right.
$$

where $n$ is the electron number density as a function of the projected distance, $n_{0}$ is the density normalization, $C$ is the density compression factor of the shock, $\alpha 1$ and $\alpha 2$ are the power-law indices, $r$ is the radial distance from the center, and $r_{\mathrm{sh}}$ is the radius corresponding to the putative edge or cold/ shock front. All the parameters of the model were allowed to vary during the fit.

The best-fit broken power-law density model parameters are summarized in Table 4 . In order to determine the nature of the detected edges at A, B (i.e., shocks or cold fronts), we need to measure temperatures on their either sides. First, we extract the spectrum from two regions $\left(\mathrm{A}_{1}\right.$ and $\left.\mathrm{A}_{2}\right)$ on either sides of edge $\mathrm{A}$ and fit them with a single temperature APEC (Smith et al. 2001) model by keeping redshift fixed at 0.032 . The best-fit temperature values for $\mathrm{A}_{1}$ and $\mathrm{A}_{2}$ are $2.39 \pm 0.40 \mathrm{keV}$ and $1.68 \pm 0.05 \mathrm{keV}$, respectively. The $\mathrm{A}_{2}$ region contains cool, dense gas and a sharp boundary. The derived temperatures from regions $A_{1}$ and $A_{2}$ are inconsistent with each other and indicate that the edge $\mathrm{A}$ is due to the presence of a cold front and is consistent with the cold front reported by Trinchieri et al. (2007).

We also extract the spectra on either side of the edge B and fit them in the same way, yielding best-fit temperatures of $2.36 \pm 0.23 \mathrm{keV}$ and $1.66 \pm 0.04 \mathrm{keV}$, respectively. These derived temperature values seem to indicate that the edge $B$ is also a cold front and consistent with the cold front reported by Trinchieri et al. (2007).

\section{Results and Discussion}

\subsection{Cooling Parameters}

Cooling time is an important parameter to understand thermal evolution of the hot baryonic matter, in an individual galaxy, or in a group or cluster of galaxies. We derived the cooling time profile of the X-ray surface brightness distribution in this group by performing a projected spectral analysis. The cooling time was then determined for each of the annular regions, in a projected sense, using the derived values of the projected electron densities and temperatures. The cooling time 

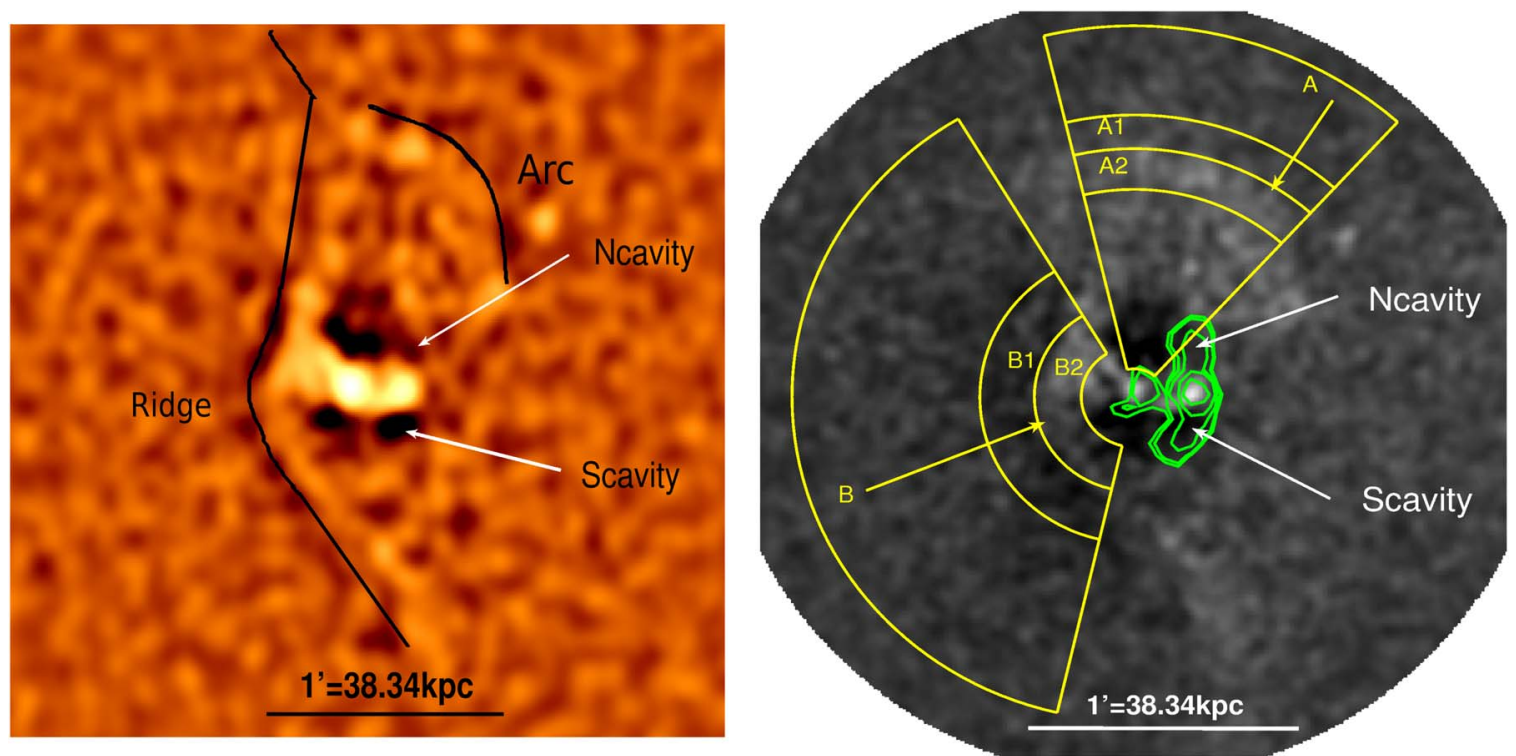

Figure 3. The $0.7-2.0 \mathrm{keV}$, unsharp-masked (left panel) and $2 \mathrm{D}$ model-subtracted, residual (right panel) images of IC 1262 . The unsharp-masked image was derived by subtracting a $10 \sigma$ wide Gaussian kernel-smoothed image from that smoothed with a $2 \sigma$ wide Gaussian, while the smooth 2D model generated from the ellipse fitting was subtracted from the original image to derive its residual map. The VLA $1.4 \mathrm{GHz}$ (green) contours are overlaid on the residual image. The contours are at $(2.5 \sigma, 3.0 \sigma, 3.5 \sigma)$ levels where $\sigma$ is $6 \mu \mathrm{Jy}$ beam $^{-1}$ for the VLA B-configuration. The annular regions used to extract the surface brightness profiles (A) $\left(30^{\circ}-110^{\circ}\right)$ and $(\mathrm{B})\left(120^{\circ}-270^{\circ}\right)$ are shown in yellow).
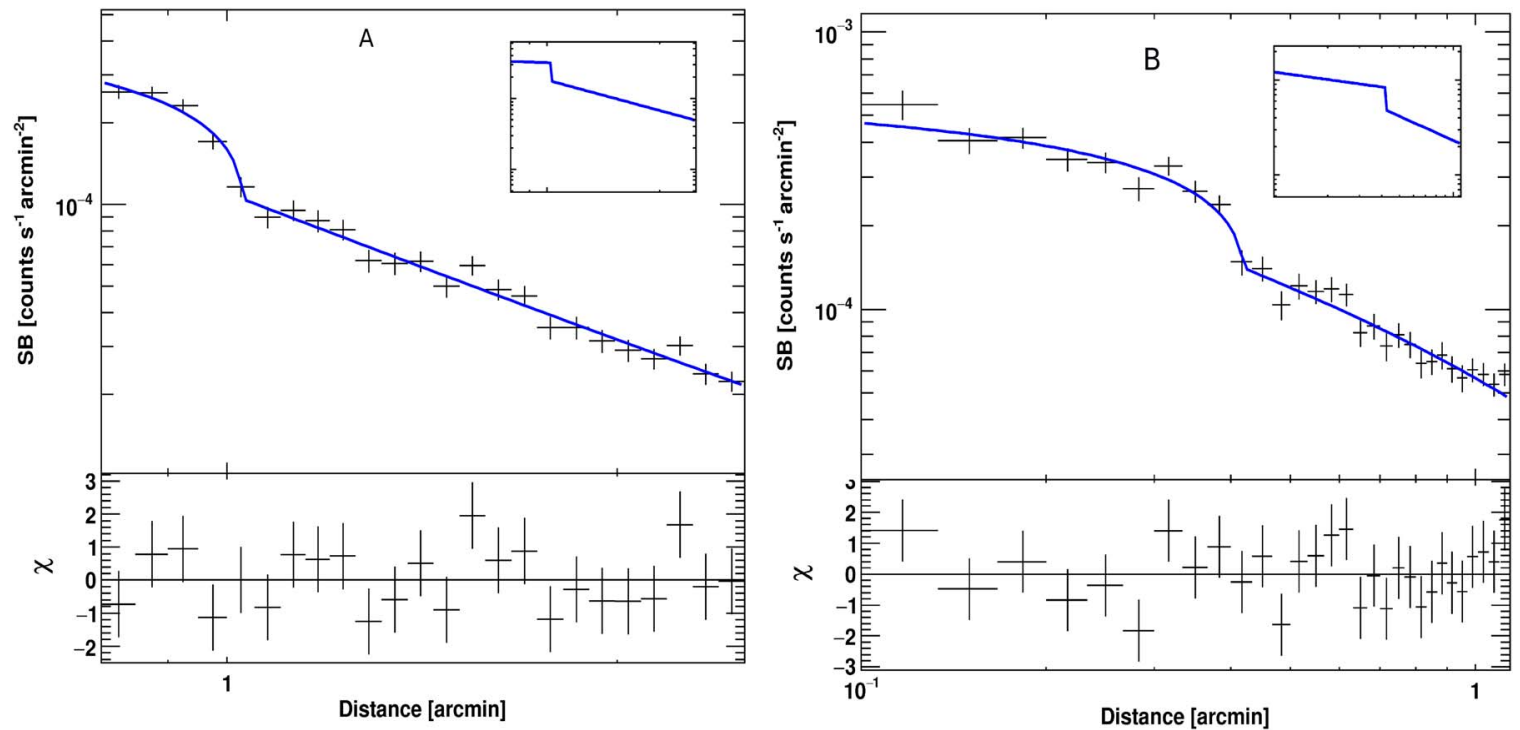

Figure 4. Projected radial surface brightness profile, in the energy range of $0.7-4.0 \mathrm{keV}$, extracted from the northwest wedge shaped sector with opening angles $\left(30^{\circ}-110^{\circ}\right)$ (left panel) and from the east region with angle $\left(120^{\circ}-270^{\circ}\right.$ ) (right panel). The surface brightness profiles are extracted from the annuli A and B, shown in yellow, in Figures 3(A), (B). In each panel we show the corresponding 3D gas density model, while the bottom panels show the residuals from the best-fit surface brightness profiles.

Table 3

X-Ray Cavity Parameters

\begin{tabular}{llccc}
\hline \hline Group & Cavity & $\begin{array}{c}a \\
(\mathrm{kpc})\end{array}$ & $\begin{array}{c}b \\
(\mathrm{kpc})\end{array}$ & $\begin{array}{c}R \\
(\mathrm{kpc})\end{array}$ \\
\hline IC 1262 & Ncavity & 2.22 & 1.52 & 6.48 \\
& Scavity & 4.01 & 2.00 & 6.13 \\
\hline
\end{tabular}

profile was then derived using Sarazin (1988):

$$
t_{\text {cool }}=8.5 \times 10^{10} \mathrm{yr}\left[\frac{n_{\mathrm{e}}}{10^{-3} \mathrm{~cm}^{-3}}\right]^{-1}\left[\frac{T_{\mathrm{gas}}}{10^{8} \mathrm{~K}}\right]^{1 / 2},
$$

where $n_{e}$ represents the electron density and $T_{\text {gas }}$ gas temperature. The resultant cooling time profile is shown in Figure 5, which reveals that the cooling time of the gas in the core of this group is much shorter than the Hubble time; in perfect agreement with those seen in many other groups (Peterson \& Fabian 2006; Giacintucci et al. 2008; McDonald et al. 2010; Panagoulia et al. 2014). This strongly supports the argument that heating by the central AGN occurs at least over a timescale of $10^{8} \mathrm{yr}$.

\subsection{X-Ray Properties within the Cooling Radius}

To investigate the global properties of the $\mathrm{X}$-ray emitting gas, we extracted a combined spectrum, from within the 
Table 4

Best-fit Parameters of the Broken Power-law Density Model

\begin{tabular}{|c|c|c|c|c|c|c|}
\hline Regions & $\alpha 1$ & $\alpha 2$ & $\begin{array}{c}r_{\mathrm{sh}} \\
(\operatorname{arcmin})\end{array}$ & $\begin{array}{c}n_{0} \\
\left(10^{-4}\right)\end{array}$ & $C$ & $\chi^{2} /$ dof \\
\hline $\mathrm{A}\left(30^{\circ}-110^{\circ}\right)$ & $0.18 \pm 0.11$ & $1.43 \pm 0.20$ & $1.02 \pm 0.02$ & $3.20 \pm 0.40$ & $1.81 \pm 0.17$ & $18.93 / 18$ \\
\hline B $\left(120^{\circ}-260^{\circ}\right)$ & $0.23 \pm 0.08$ & $1.30 \pm 0.02$ & $0.41 \pm 0.03$ & $6.60 \pm 0.03$ & $1.52 \pm 0.02$ & $48.96 / 48$ \\
\hline
\end{tabular}

cooling radius ( $\leqslant r_{\text {cool }} 80 \mathrm{kpc}$ ), in the energy range $0.7-8.0 \mathrm{keV}$ for this group. The cooling radius is the radius within which gas cools faster than $7.7 \times 10^{9} \mathrm{yr}$, the time in which the cluster/group is believed to relax and establish a cooling flow.

The spectrum was extracted using the CIAO tool SPECEXTRACT and grouped to have a minimum of 25 counts per spectral bin. This was done after removing compact point-like sources, including the central $\sim 2^{\prime \prime}$ region. The count-weighted response matrices were generated for each of the extractions. The extracted spectrum was then exported to the fitting package XSPEC and fitted with the model (wabs $\times$ apec). The gas temperature $k T$ and APEC normalization $N$ were allowed to vary during the fit. We repeated the fitting exercise by freeing and fixing the values of the Galactic hydrogen column $N_{\mathrm{H}}$ and the gas abundance $Z$. The fit in which $N_{\mathrm{H}}$ and $Z$ were allowed to vary returned in the best-fit results. Using these best-fit parameters, we derive the $0.7-10.0 \mathrm{keV} \mathrm{X}$-ray luminosity, from within the cooling radius $\left(L_{\text {cool }}\right)$ for the IC 1262 group, to be equal to $3.29_{-0.3}^{+0.2} \times 10^{42} \mathrm{erg} \mathrm{s}^{-1}$.

\subsection{X-Ray and Radio Morphology}

In order to map the diffuse radio emission and its extent in this group, we generate a tricolor map, using GMRT radio, VLA radio, and Chandra X-ray observations, shown in Figure 6. In this figure, red denotes GMRT $325 \mathrm{MHz}$ radio emission, green denotes the high resolution VLA radio $1.4 \mathrm{GHz}$ emission map, and blue denotes the soft X-ray (0.7-2.0) keV emission detected by Chandra. From this figure it is evident that radio lobes are more extended than that of the $\mathrm{X}$-ray emission. The detailed spectral properties of multifrequency radio emission are discussed in Section 4.7.

\subsection{Cavity Energetics}

In the present study we assume the X-ray cavities to be bubbles devoid of gas, moving outward due to buoyant force at the local ambient temperature. Volumes of the individual cavities were calculated assuming that they are symmetric about the plane of the sky, with their centers lying in the plane perpendicular to the line of sight passing through the central AGN. For this, we assumed the cavities of prolate ellipsoidal shape, with semimajor axis $a$ and semiminor axis $b$. The volume of each cavity was estimated as $V=4 \pi a b R / 3$, where $R$ is the line-of-sight distance between the nuclear source and the center of the cavity.

Following the method proposed by Bîrzan et al. (2004), we estimate the age of the cavities as given in column 7 of Table 5. This analysis has enabled us to investigate two clear cavities with their projected distance from the center of a group equal to $\sim 6.48 \mathrm{kpc}$ and $\sim 6.13 \mathrm{kpc}$. Generally, the age derived using the approach of sound crossing provides lower estimates, while that derived from time required to refill the cavities lead to estimates on the higher side. The estimates of the total mechanical power content of the cavities for the IC 1262 group are given in column 8 of Table 5 . The total mechanical power contained in these two cavities is about $12.37 \times 10^{42} \mathrm{erg} \mathrm{s}^{-1}$.

\subsection{Quenching of the Cooling Flow}

To cross-check whether the apparent AGN feedback can efficiently work to quench the cooling flow in IC 1262, we compare the balance between the total AGN output power $\left(P_{\text {cav }}\right)$, derived from the gas luminosity within the cooling radius $L_{\mathrm{ICM}}\left(<r_{\mathrm{cool}}\right)$. This is shown in the heating versus cooling diagram (Figure 7 left panel). Here, $P_{\text {cav }}$ gives the measure of the energy injected by the AGN into the hot gas, while $L_{\mathrm{ICM}}\left(<r_{\text {cool }}\right)$ represents the energy lost by the hot gas from within the cooling radius in the form of X-ray emission. This comparison reveals that the radio source hosted by the central dominant galaxy IC 1262 is capable enough to deposit sufficient energy into the IGM to offset the cooling flow.

The equality between the heating and cooling at the heat input rates of $p V, 4 p V$, and $16 p V$ per cavity are shown by the diagonal lines in this figure. In the same figure we also plot the similar results derived by other authors (Rafferty et al. 2006; O'Sullivan et al. 2011; Pandge et al. 2013; Vagshette et al. 2016, 2017). The position of the IC 1262 group in this plot, with respect to the others from the literature, is shown by a magenta " + ," and is found to lie near the $4 p V$ enthalpy line. This in turn confirms that the total power available within the cavities is sufficient to offset the cooling flow within the cooling radius.

We also compared the $P_{1.4} \mathrm{GHz}$ radio power with total cavity power $\left(P_{\text {cav }}\right)$ (Figure 7 , right panel). In this figure, filled circles represent the sample studied by Cavagnolo et al. (2010), and IC 1262 is marked by a magenta "+" symbol. This plot also indicates that the radio source associated with IC 1262 is capable of quenching the cooling flow in this group.

\subsection{Association of X-Ray Cavities with Radio Emission}

It is well established that the X-ray surface brightness depressions seen in a majority of the cool-core systems are produced due to the interaction between the radio lobes, originating from the active nucleus, and the surrounding ICM, even in galaxy groups (Jetha et al. 2008; Giacintucci et al. 2011; Pandge et al. 2012, 2013; Vagshette et al. 2016). The radio jets emanating from the cores of the groups or clusters displaces the surrounding hot gas and leaves depressions or cavities in their surface brightness. These X-ray cavities are often found to be filled with radio emission in many of the systems.

We have attempted to examine whether the X-ray cavities in this group also have a similar association with the radio emission from the central source. For this we have made use of the multifrequency radio data available in the VLA and GMRT archives. Results from the analysis of the radio data on this system are shown in Figure 8, where panel A shows the GMRT extended radio emission map at $325 \mathrm{MHz}$, and its contours 


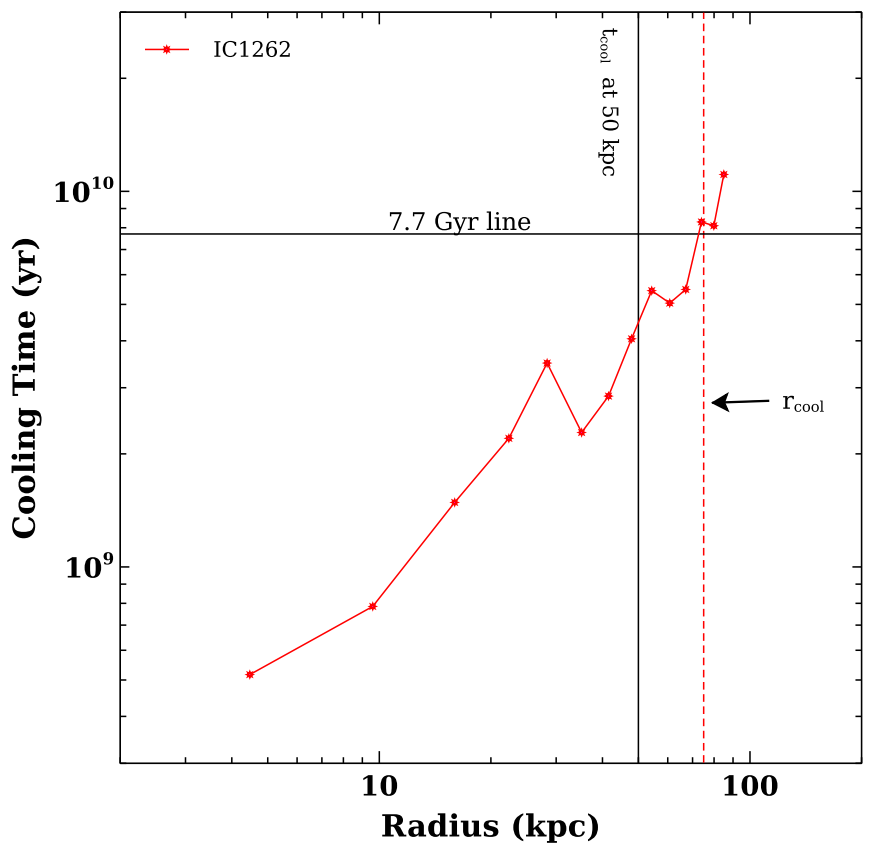

Figure 5. Cooling time profile of IC 1262. The horizontal solid line corresponds to the cooling time of $7.7 \mathrm{Gyr}$. The vertical solid line (black) represents a cooling radius at $50 \mathrm{kpc}$, while the vertical dashed line (red) represents a cooling radius of IC 1262 group where $R_{\text {cool }}=80 \mathrm{kpc}$ at $7.7 \mathrm{Gyr}$.

from the central source, while panels (B) and (C) are higher resolution images from the VLA (B array) at $1.4 \mathrm{GHz}$ with a beam size of 3 " $62 \times 3$ ". 48 and $\mathrm{rms}$ of $0.02 \mathrm{mJy} /$ beam. Image $\mathrm{B}$ shows the inner core and jet structure whereas image $\mathrm{C}$ shows the possible southern lobe that has highly unusual structure.

The morphology of the radio emission varies greatly over the images and appears to be quite complicated. Radio emission from IC 1262 appears to be widespread, extending over more than $5^{\prime}$ ( $\left.\sim 200 \mathrm{kpc}\right)$. The radio source in IC 1262 is found to be associated with both the cavities detected in the X-ray image of this group and appears to coincide with the X-ray center of this group (Figure 3, right panel). It implies the presence of two radio sources, one relatively weaker object associated with the obvious X-ray bright source, and another appearing 14".62 $(\sim 9.7 \mathrm{kpc})$ to the east of the former at about. This radio image shows a clear jet-like structure in this system (see Figure 8(B)).

We have also detected another X-ray depression at the position of the southern radio lobe, which could be the first generation $\mathrm{X}$-ray cavity and is shown by white arrows in Figure 9 (left panel). GMRT $325 \mathrm{MHz}$ contours (green color) overlaid on this image are shown in Figure 9 (right panel). An excess X-ray emission on the west to the center highlighted by white arrows is also shown in this figure. A weak shock to the south of the central source at a projected distance of $200 \mathrm{kpc}$ has been detected and is shown by a (white) dotted arc in Figure 9 (left panel). A weak shock (of Mach number 1.5) has been reported at this location by Sun et al. (2009). We could not detect a first generation cavity at the position of the northern lobe.

\subsection{Spectral Index of IC 1262}

\subsubsection{Spectral Plot}

We measured flux densities for three different regions of IC 1262-(1) the North Lobe (NL), (2) the Central Source (CS),

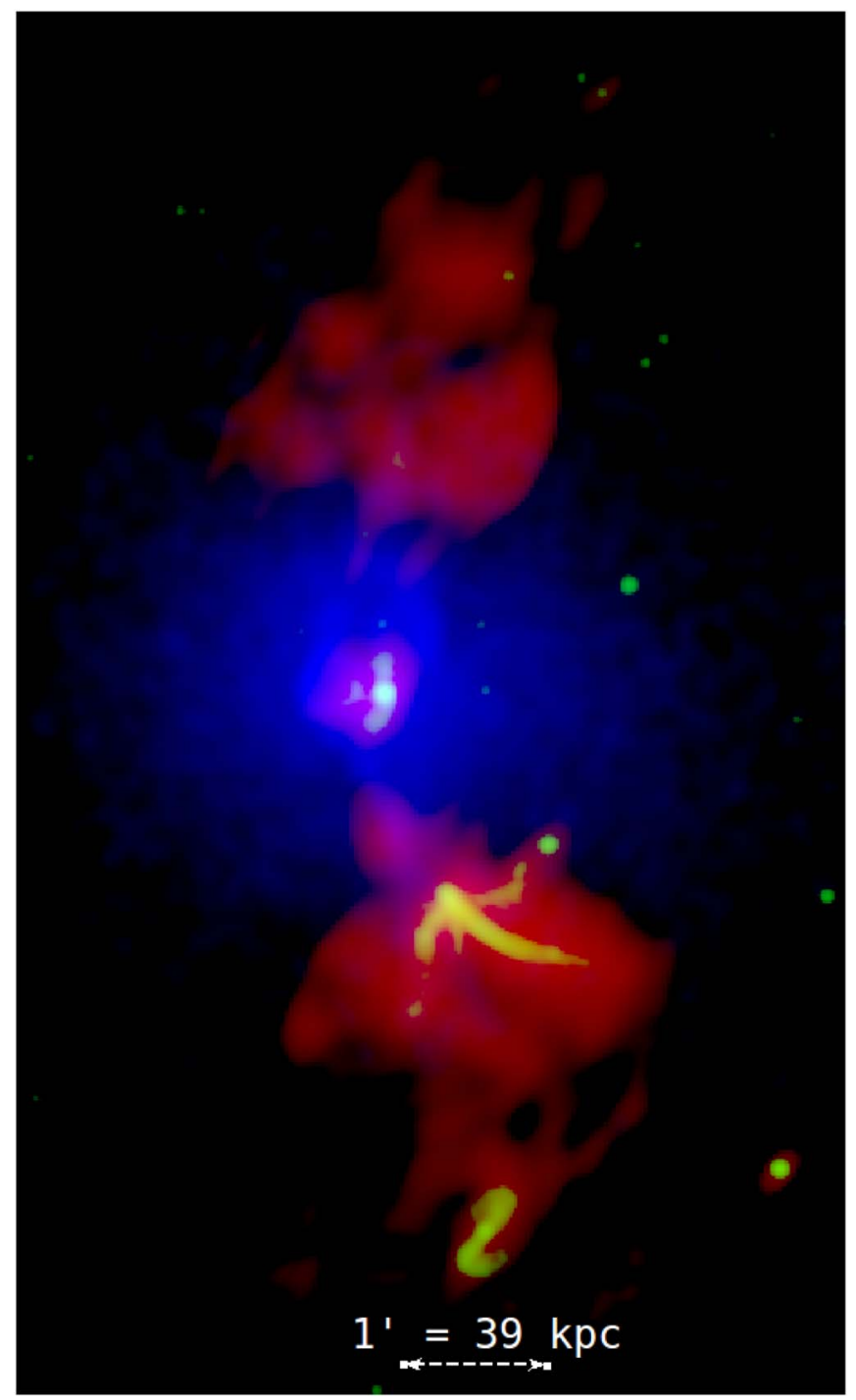

Figure 6. Tricolor image of IC 1262 , where red denotes $325 \mathrm{MHz}$ radio emission (GMRT), green denotes $1.4 \mathrm{GHz}$ radio emission at high resolution (JVLA), and blue denotes soft X-ray (0.7-2.0) keV emission from Chandra.

and (3) the South Lobe (SL) using observations from the $150 \mathrm{MHz}$ TIFR-GMRT-Sky Survey (TGSS), VLA $P$ (325 MHz), and $L(1400 \mathrm{MHz})$ bands.

In the $L$-band, D-configuration image, we see two separate radio sources on the south of the central radio source, which are blended in both the lower resolution $P$ (VLA) and TGSS images. In the flux density calculation, we separate out this southern source (R.A.: $17^{\mathrm{h}} 32^{\mathrm{m}} 58^{\mathrm{s}}$, decl.:43 $41^{\prime} 45^{\prime \prime}$ ) from that in the South lobe (R.A.: $17^{\mathrm{h}} 32^{\mathrm{m}} 59^{\mathrm{s}}$, decl.:43 $43^{\prime} 44^{\prime \prime}$ ). We have listed our measurements (deconvolved sizes and integrated flux densities) in Table 6.

To calculate the flux densities of these sources, we convolved the $L$-band (resolution of $41^{\prime \prime} \times 37^{\prime \prime}$ ) and TGSS (resolution of $20^{\prime \prime} \times 20^{\prime \prime}$ ) images to $P$ (VLA) band image of beam size of $57^{\prime \prime} \times 41^{\prime \prime}$. The rms in the $L$-band, $P$-band, and TGSS images are $0.15 \mathrm{mJy}$ beam $^{-1}, 1.5 \mathrm{mJy}$ beam $^{-1}$ and $20 \mathrm{mJy} \mathrm{beam}^{-1}$, respectively. We scaled TGSS, $P$-, and $L$-band flux density values to the recent (Perley \& Butler 2017) flux density scale. This new flux density scale provides accurate measurements over $50 \mathrm{MHz}-$ $50 \mathrm{GHz}$ using current observations with the Jansky VLA. We 

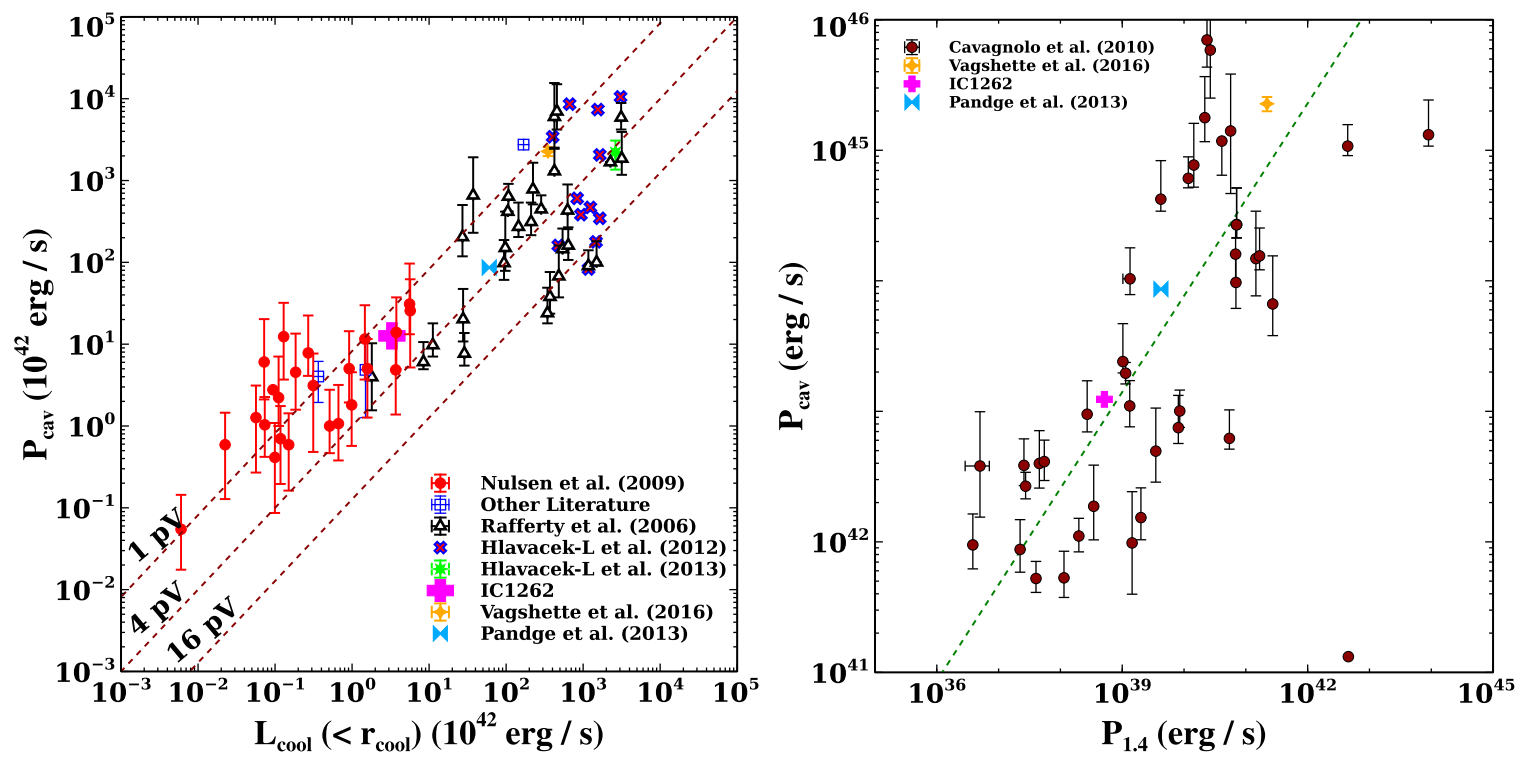

Figure 7. Left panel: cavity power vs. X-ray luminosity within the cooling radius of groups and clusters (from Rafferty et al. 2006), represented as filled circles (black). The cavity power and X-ray luminosity for IC 1262 is shown by the "+" symbol (magenta). The diagonal lines represent samples where $P_{\text {cav }}=L_{\mathrm{ICM}}$, assuming $p V, 4 p V, 16 p V$ as the total enthalpy of the cavities. Right panel: cavity power plotted against radio power, with filled circles representing galaxy clusters and groups from Cavagnolo et al. (2010). The magenta “+” represents the IC 1262 group.

Table 5

Cavity Parameters from the X-Ray Observations

\begin{tabular}{|c|c|c|c|c|c|c|c|}
\hline $\begin{array}{l}\text { Group } \\
\text { (1) }\end{array}$ & $\begin{array}{l}\text { Cavity } \\
\text { (2) }\end{array}$ & $\begin{array}{c}E_{\text {bubble }}=p V \\
\text { (3) } \\
10^{56} \mathrm{erg}\end{array}$ & $\begin{array}{c}t_{\mathrm{c}_{s}} \\
(4) \\
10^{7} \mathrm{yr}\end{array}$ & $\begin{array}{c}t_{\text {buoy }} \\
(5) \\
10^{7} \mathrm{yr}\end{array}$ & $\begin{array}{c}t_{\text {refill }} \\
(6) \\
10^{7} \mathrm{yr}\end{array}$ & $\begin{array}{c}t_{\text {avg }} \\
(7) \\
10^{7} \mathrm{yr}\end{array}$ & $\begin{array}{c}P_{\text {cav }} \\
(8) \\
10^{42} \mathrm{erg} \mathrm{s}^{-1}\end{array}$ \\
\hline \multirow[t]{2}{*}{ IC 1262} & Ncavity & 58.0 & 1.7 & 2.4 & 5.2 & 3.1 & 6.0 \\
\hline & Scavity & 50.1 & 1.2 & 2.1 & 4.2 & 2.5 & 6.3 \\
\hline
\end{tabular}

Note. Column 3: total energy stored in each of the cavities. Columns 4, 5, and 6: cavity ages estimated in three different ways (see the text). Column 7: average age of cavities. Column 8: power stored in each cavity.

plotted the integrated spectra of IC 1262 in Figure 10, where both the lobes exhibit steep spectrum radio sources (we follow the convention $S \propto \nu^{\alpha}$, where $\alpha$ is the spectral index and $S$ is the flux density at frequency $\nu$ ). We find that the radio luminosity of the south lobe exceeds that of the north lobe at all these frequencies. This is also in agreement with the spectral index of the north lobe $(\alpha 1=-2.23)$ and is steeper than the south lobe $(\alpha 3=-1.94)$. Moreover, we find that average spectral index values for IC 1262 radio galaxy, including and excluding the central AGN, are 1.73 and 2.08 , respectively.

The error estimation in the flux density measurements were carried out using the following procedure. There are two primary sources of errors in the flux density measurements: (1) one due to the uncertainties in the flux densities of the unresolved source(s) used for the calibration of the data. We assumed this error to be $\sim 10 \%$ at 325 and $1400 \mathrm{MHz}$, while that at $150 \mathrm{MHz}$ to be $20 \%$; (2) because these radio sources (the north and the south radio lobes) are extended sources, the errors in their flux density estimations will be the rms in the image multiplied by the square root of the ratio of the solid angle of the source to that of the synthesized beam. Since these two sources of errors are unrelated, we added them in quadrature to estimate the final error on the flux densities of the extended sources, using

$$
\left.\Delta S=\left[\left(\sigma_{\mathrm{amp}} S\right)^{2}+\left(\sigma_{\mathrm{rms}} \sqrt{n_{\mathrm{beams}}}\right)^{2}\right)\right]^{1 / 2},
$$

where $S$ is the flux density, $\sigma_{\text {rms }}$ is the image rms noise, and $n_{\text {beams }}$ is the number of beams within the extent of the source.

\subsubsection{Spectral Index Maps}

Three spectral index maps were made, combining data from VLA, GMRT, and TGSS, as shown in Figure 11. The maps at various frequencies were convolved appropriately according to the requirements as described below.

1. The higher resolution (16". $40 \times 42$ ". 13$)$ GMRT $325 \mathrm{MHz}$ map was convolved to the beam size of the TGSS map $\left(25^{\prime \prime} \times 25^{\prime \prime}\right)$. This is shown in Figure 11(A).

2. The GMRT $325 \mathrm{MHz}$ map was convolved to the beam size of the VLA L-band (D-configuration) map $(40$ ". $96 \times 37$ !"00), as shown in Figure 11(B).

3 . The high resolution $(3$ !" $63 \times 3$ !" 28$)$ VLA $L$-band (B-configuration) map was convolved to the resolution of GMRT $325 \mathrm{MHz}$ map, as shown in Figure 12(A).

After the above procedure, the routine tasks in AIPS (OHGEO and COMB) were used to make the spectral index maps. Pixels below $3 \sigma$ rms were blanked in images $\mathrm{A}$ and $\mathrm{B}$ before computing the spectral index maps. For Figure 12(A), a higher cutoff of $5 \sigma \mathrm{rms}$ was observed for higher precision. In Figure 12(A), the contours of the VLA L-band (B configuration) are overlaid with its beam size represented in the bottom 
(A)

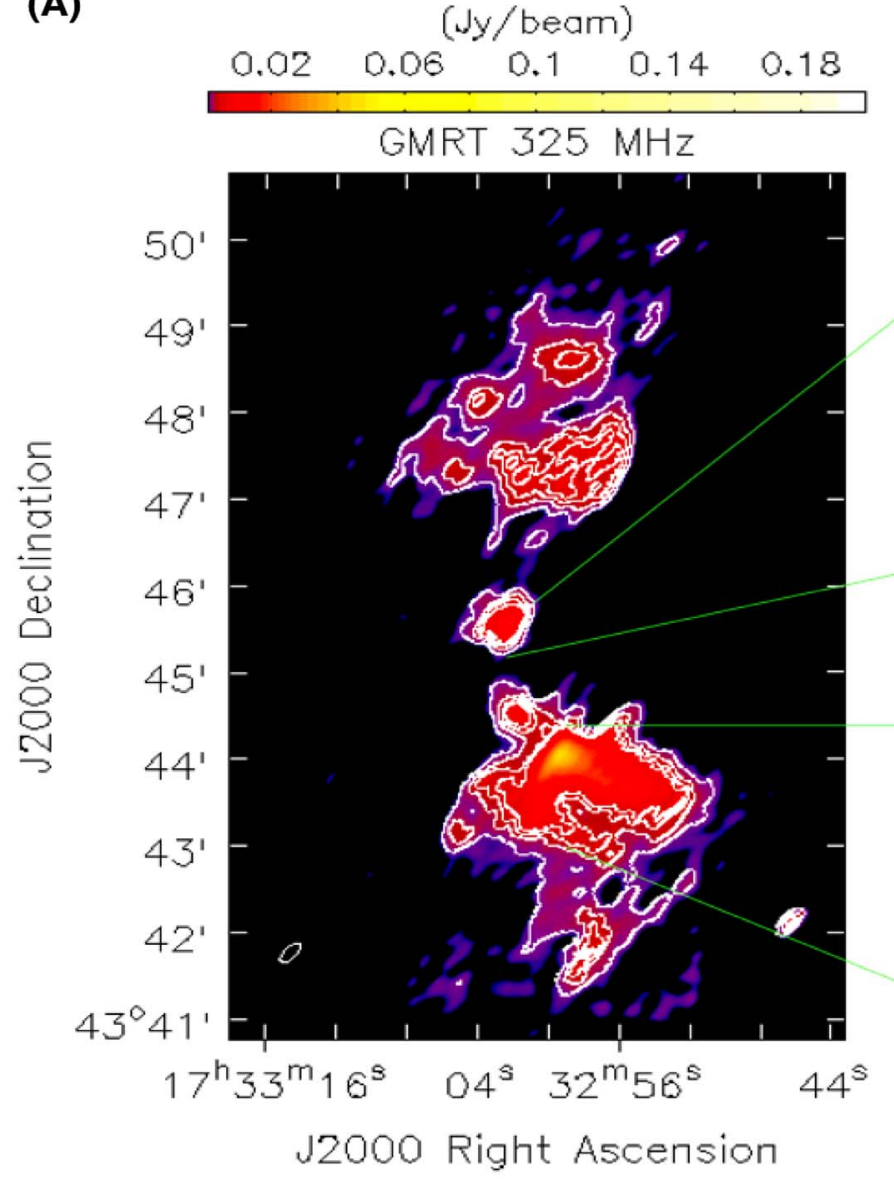

(B)

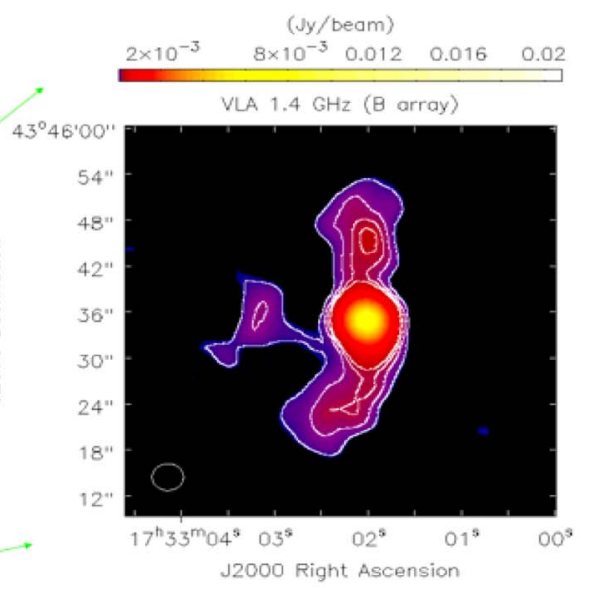

(C)
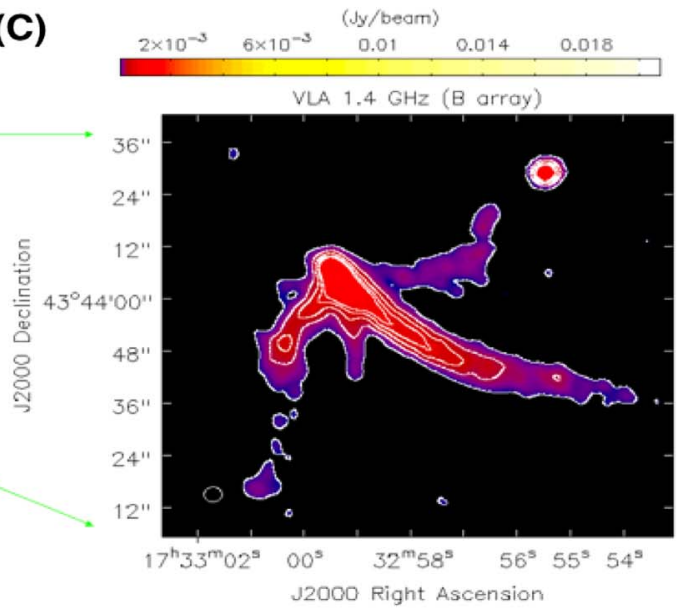

Figure 8. Color and contour maps of IC 1262 made, using the task "viewer" in CASA with relative contour levels at $[0.2,0.4,0.6,0.8] \mathrm{mJy} / \mathrm{beam}$. Image (A) shows the GMRT $325 \mathrm{MHz}$ data with a beam size of 16 ". $40 \times 7$ !' 39 , where we can see the entire radio source at rms of $\sim 0.25 \mathrm{mJy} /$ beam. On the right-hand side of the image, we see the zoomed-in version of the various components of the source. Images (B) and (C) are higher resolution images from JVLA (B-array) at $1.4 \mathrm{GHz}$ with a beam size of 3 !" $62 \times 3$ !" 48 and rms of $0.02 \mathrm{mJy} /$ beam. Image B shows the inner core and jet structure. Image C shows the southern lobe of unusual structure.
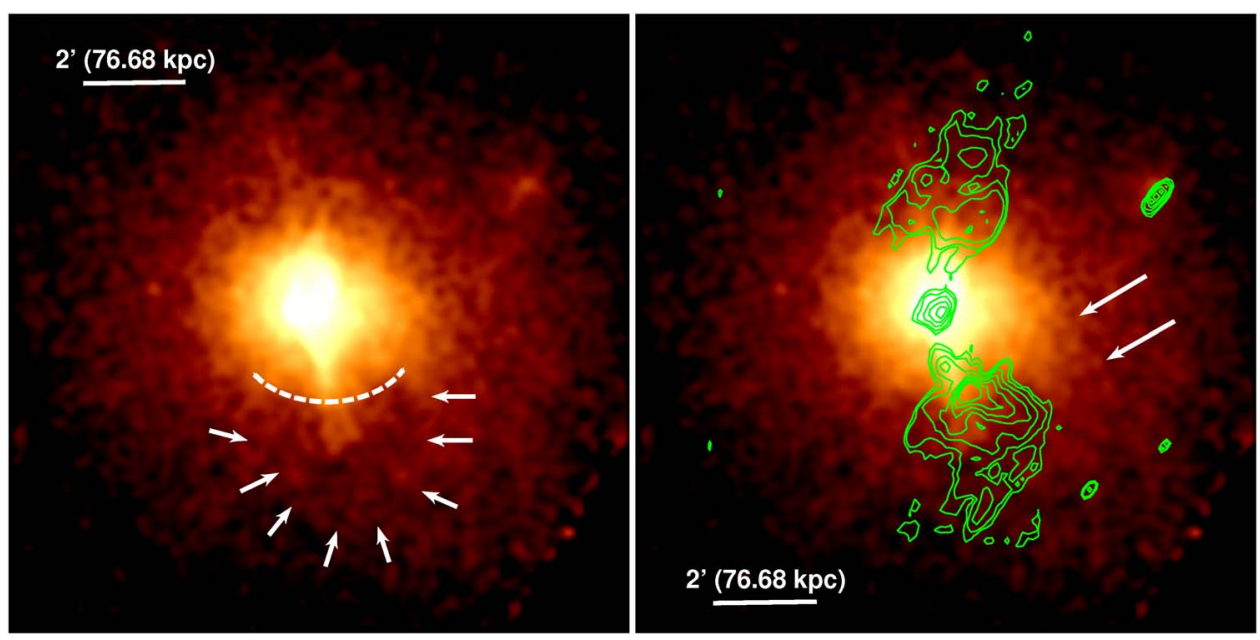

Figure 9. Left panel: the Chandra image of IC 1262, corrected for exposure and background, in the energy range $0.7-7.0 \mathrm{keV}$. This X-ray image is smoothed by a $6 \sigma$-wide Gaussian kernel $(\sigma=1$ pixel). The location of a first generation X-ray depression is shown by white arrows, while the position of the shock is shown by a white dotted arc. Right panel: same figure as in the left panel, on which GMRT $325 \mathrm{MHz}$ radio contours are overlaid. The excess X-ray emission seen southwest of the central source is highlighted by white arrows.

left corner of the image. From these figures it is evident that the average spectral index obtained for the northern lobe is $\sim-2.23$, and that of the southern lobe is $\sim-1.94$ (see
Figure 10). Figures 10 and 11 clearly show that the average spectral index obtained for the northern lobe is steeper than that of the southern lobe. The average spectral index of both the 
Table 6

Radio Parameters

\begin{tabular}{|c|c|c|c|c|c|}
\hline Region & $\begin{array}{c}\text { Frequency } \\
\mathrm{MHz}\end{array}$ & $\begin{array}{l}\text { Flux Density } \\
\text { mJy }\end{array}$ & $\begin{array}{l}\text { Angular Size (deconvolved) } \\
\qquad \times "\end{array}$ & $\begin{array}{l}\text { Linear Size } \\
\mathrm{kpc} \times \mathrm{kpc}\end{array}$ & $\begin{array}{c}P_{1.4 \mathrm{GHz}} \\
10^{23}\left(\mathrm{~W} \mathrm{~Hz}^{-1}\right)\end{array}$ \\
\hline \multirow[t]{2}{*}{ North Lobe (NL) } & 150 & $1135 \pm 4.6$ & $113 \times 90$ & $68 \times 54$ & \\
\hline & 325 & $341.2 \pm 0.25$ & $97 \times 86$ & $58 \times 22$ & \\
\hline \multirow[t]{2}{*}{ Central Source (CS) } & 150 & $418.3 \pm 4.5$ & $86 \times 37$ & $51 \times 22$ & \\
\hline & 325 & $112.1 \pm 0.25$ & $78 \times 24$ & $47 \times 14$ & \\
\hline \multirow[t]{3}{*}{ South Lobe (SL) } & 150 & $2140 \pm 4.6$ & $92 \times 75$ & $55 \times 45$ & \\
\hline & 325 & $855.1 \pm 0.25$ & $76 \times 71$ & $46 \times 43$ & \\
\hline & 1400 & $50.01 \pm 0.06$ & $53 \times 44$ & $32 \times 27$ & 1.0 \\
\hline
\end{tabular}

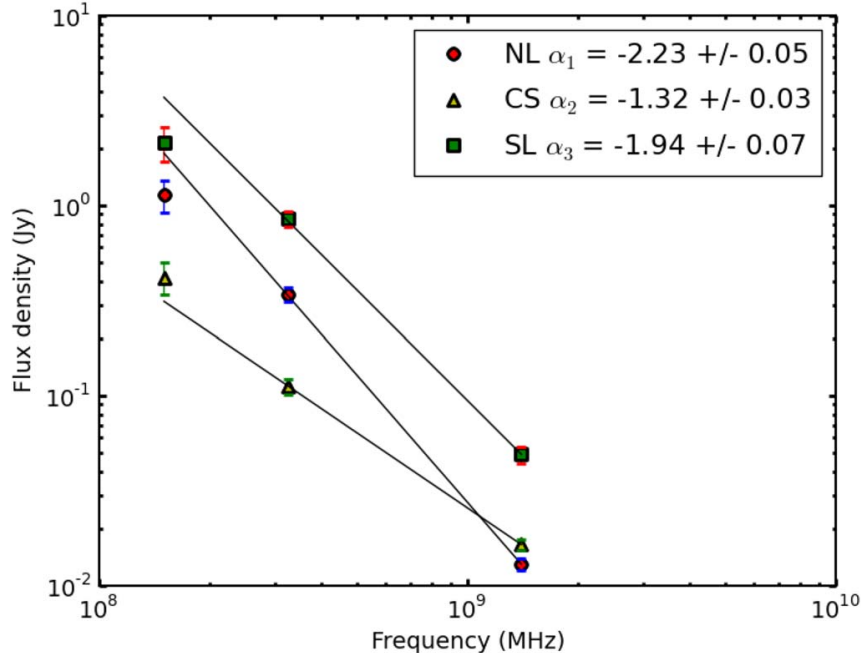

Figure 10. Integrated radio spectrum of IC 1262.

lobes is extremely steep relative to that found in typical low redshift radio galaxies, namely $\sim 0.7$ (Blundell et al. 1999).

\subsubsection{Radio Phoenix in the Southern Radio Lobe}

Extended radio sources found in galaxy clusters and groups are interpreted in a variety of ways, in terms of their inferred age and origin, from observed parameters such as extent, shape, flux, and spectral index. A taxonomy of such sources has been summarized in Kempner et al. (2004), in which a radio phoenix appears as an extended filamentary steep spectrum (spectral index -1.5) radio source. These are found in the cores of groups and clusters, and are interpreted as an indication of a recent merger. Accretion shocks in mergers of groups and clusters can be found over regions as large as hundreds of kiloparsecs. These appear extended more frequently at lower than at gigahertz frequencies. Radio phoenixes start their lives as relics resulting from an earlier merger and have a population of charged particles that have aged significantly such that their synchrotron radiation is too faint to detect at high frequencies (e.g., Enßlin \& Gopal-Krishna 2001). The accretion shock due to a recent merger then rejuvenates this faded relic, by compressing the plasma and reaccelerating the particles to energies such that they would again be detectable at radio frequencies. the analogy is thus to a phoenix rising from its embers. The classic example of a radio phoenix is the cluster Abell 85 (Young et al. 2005).

The complex feature detected in the southern radio lobe (Figure $8(C)$ ) is not associated with any X-ray or optical emission, but is found to be embedded within the southern radio lobe, suggesting that it is related to the central radio AGN. The radio emission is complex and largely filamentary in morphology, which usually indicates interaction with the surrounding ICM, and is characteristic of a phoenix. The average value of the radio spectral index of this feature within the southern radio lobe (Figure 8(C), obtained by combining fluxes from TGSS, GMRT $325 \mathrm{MHz}$, and VLA $L$-band in the B-configuration) is equal to $\sim-1.92$. As noted above, this is consistent with that of a phoenix, where the aged plasma would have been compressed adiabatically by merger shock waves boosting the radio emission (Enßlin \& Gopal-Krishna 2001; Kempner et al. 2004; Ogrean et al. 2011; Van Weeren et al. 2012).

Several radio phoenixes with these characteristic features have been identified so far, but they have all been discovered in rich clusters with high mass or X-ray temperature. Prominent examples are Abell 85 (radial velocity dispersion $=692 \mathrm{~km} \mathrm{~s}^{-1}$ ) and Abell 1033 (radial velocity dispersion $=677 \mathrm{~km} \mathrm{~s}^{-1}$, both values taken from Rines et al. 2016). However, IC 1262 is considered to be a poor group, its radial velocity dispersion being about $=300 \mathrm{~km} \mathrm{~s}^{-1}$ (Wegner et al. 1999), and thus we find that this is the first case where such an object has been found in a group-scale dark halo, showing that merger shocks can revive relic plasma in group-scale mergers as well.

We also checked the variation of the spectral index along this complex structure, computed using the regions shown in the inset (Figure 12(B)). This shows that the spectrum of the diffuse emission steepens with increasing distance from the peak of the radio emission, which indicates that plasma overall has aged and lost its energy via synchrotron or inverse Compton (IC) radiation as it has moved away from its source. However, the feature in question, appears to have been revived by the passing of a weak merger shock with a Mach number $\mathcal{M} \sim 1.2$ (Sun et al. 2009), sufficient for compressing the aged radio plasma.

To find the possible merger signature in the $\mathrm{X}$-ray image described above, we postulate that the complex filamentary structure within the southern radio lobe originates from an aged radio lobe due from the central radio $\mathrm{AGN}$, which has been "revived" by a weak merger shock passing through this galaxy group. Thus, we classify this complex feature as a radio phoenix (Ogrean et al. 2011). 


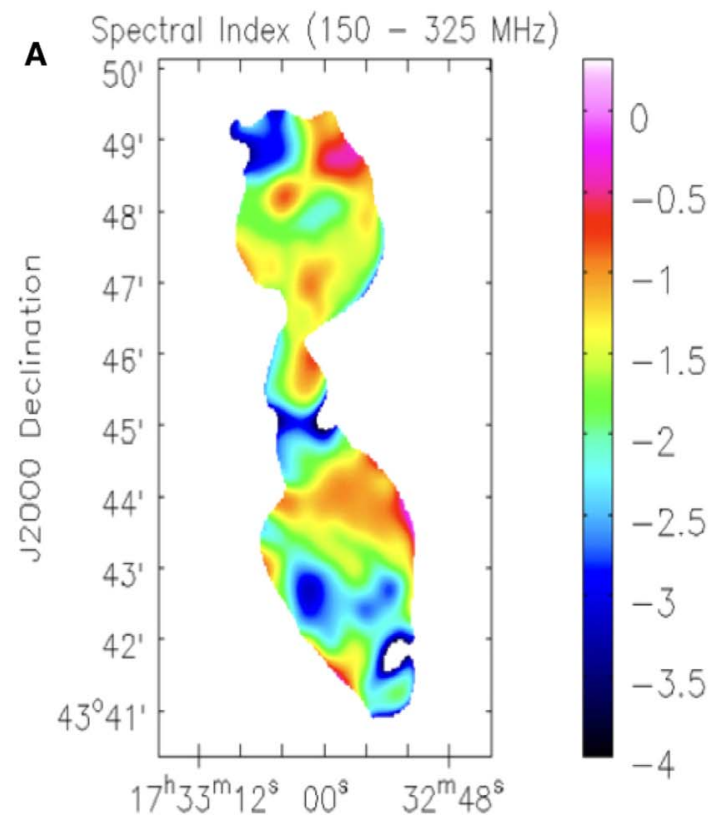

J2000 Right Ascension

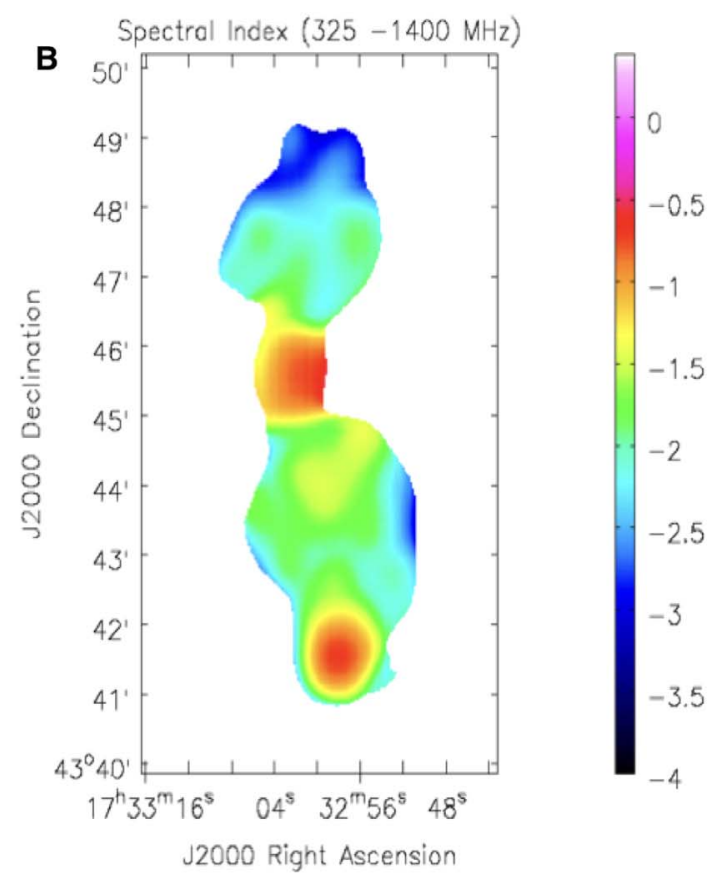

Figure 11. (A) Spectral index map between $150 \mathrm{MHz}$ (TGSS) and $325 \mathrm{MHz}$ (GMRT) frequencies. (B) Spectral index map between $325 \mathrm{MHz}$ (GMRT) and $1400 \mathrm{MHz}$ (JVLA) frequencies very clearly depicting the steep regions.

A

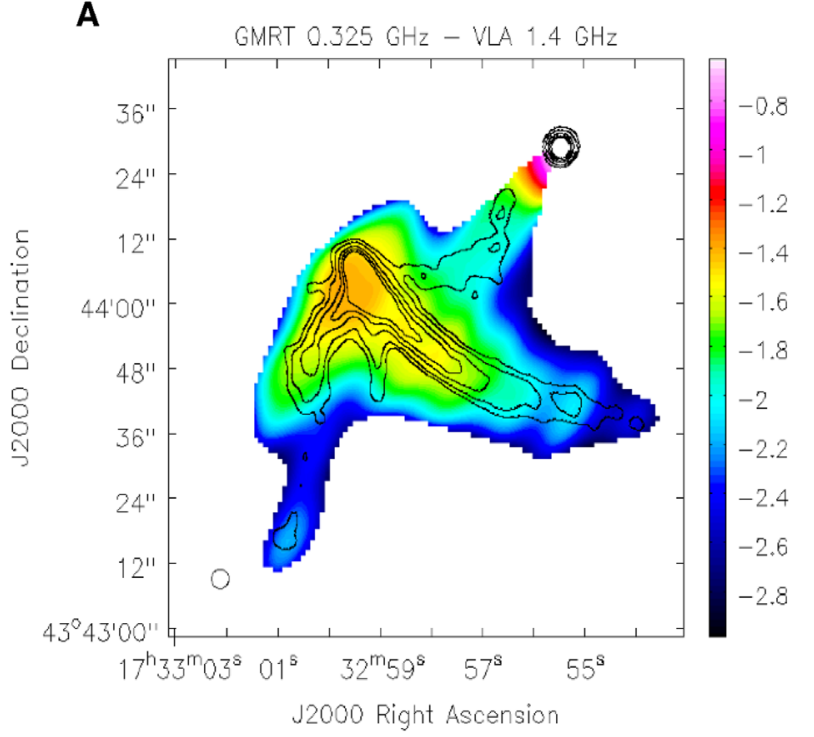

B

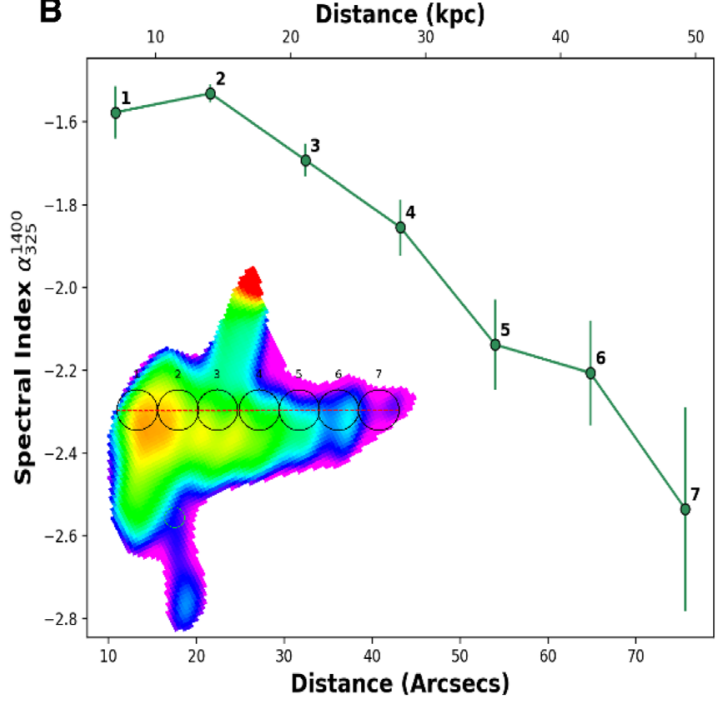

Figure 12. (A) The spectral index map of the radio phoenix, measured between $325 \mathrm{MHz}$ (GMRT) and $1400 \mathrm{MHz}$ (VLA) frequencies. (B) Spectral index along the radio phoenix, which is embedded in the southern radio lobe of IC 1262, computed using the regions shown in the inset.

\section{Conclusions}

We present results based on the analysis of 120 ks Chandra X-ray, SDSS optical, VLA 350/1400 GHz, and GMRT 235/ $610 \mathrm{MHz}$ radio observations of the galaxy group IC 1262 . The objectives of this study were to identify and confirm the positions of the X-ray cavities and surface brightness edges present in the hot IGM of IC 1262 . We summarize the important results derived from the present analysis:

1. In the unsharp masked as well as the $2 \mathrm{D} \beta$-modelsubtracted residual images of the hot IGM, we find two
X-ray cavities (Ncavity and Scavity) and a ridge around the center of the group IC 1262.

2. The X-ray cavities are located at projected distances of $\sim 6.48 \mathrm{kpc}$ and $\sim 6.13 \mathrm{kpc}$ from the center of IC 1262 .

3. Two surface brightness edges are evident to the east and northwest of the center of this group and confirmed as cold fronts.

4. The total mechanical power of both X-ray cavities $L_{\text {Cavity }}$ and the X-ray luminosity within the cooling radius $L_{\text {cool }}$ indicate that the total mechanical power emitted by the 
central radio source is sufficient to balance the cooling loss in this group.

5. The radio emission from the Jansky VLA $1400 \mathrm{MHz}$ observation appears to coincide with the location of detected X-ray cavities.

6. The analysis of the X-ray cavity images and the estimated cooling time enabled us to calculate the mechanical power for the cavities to be $P_{\text {cavity }} \sim 12.37 \times 10^{42} \mathrm{erg} \mathrm{s}^{-1}$ and $L_{\text {cool }} \sim 3.29 \times 10^{42} \mathrm{erg} \mathrm{s}^{-1}$, respectively. The comparison of these values implied that the radio jet-mode feedback is sufficient to quench the cooling losses occurring in this group within the cooling radius.

7. From the radio imaging analysis, and the spectral index plot and maps, it is evident that the radio sources hosted by IC 1262 have a flatter spectrum at the core, while that at the lobes appears steeper.

8. The radio galaxy belonging to the IC 1262 group is the low-redshift ultra-steep radio galaxy detected with a spectral index $\alpha \sim-1.73$ and $\alpha \sim-2.08$ with and without the central AGN, respectively.

9. The X-ray depression at the position of southern radio lobe has been detected in the present analysis. It is likely that it represents a first generation X-ray cavity.

10. We detect a radio phoenix embedded within the southern radio lobe, for the first time in a poor group, having a spectral index $(\alpha \leqslant-1.92)$. Its spectral index steepens with increasing distance from its peak.

M.B.P. gratefully acknowledges support from the following funding schemes: the Department of Science and Technology (DST), New Delhi under the SERB Young Scientist Scheme (sanctioned No: SERB/YSS/2015/000534), the Department of Science and Technology (DST), New Delhi under the INSPIRE faculty Scheme (sanctioned No: DST/INSPIRE/04/ 2015/000108). M.B.P. wishes to acknowledge with thanks the support received from IUCAA, India, in the form of a visiting associateship. P.D. gratefully acknowledges generous support from the Indo-French Center for the Promotion of Advanced Research (center Franco-Indien pour la Promotion de la Recherche Avan cee) under programme No. 5204-2. The authors gratefully acknowledge the use of computing and library facilities of the Inter-University center for Astronomy and Astrophysics (IUCAA), Pune, India. This research work has made use of data from the Chandra Data Archive, NASAs Astrophysics Data System (ADS), NASA/IPAC Extragalactic Database (NED), High Energy Astrophysics Science Archive Research Center (HEASARC), and softwares CIAO, ChIPS, and Sherpa provided by the Chandra X-ray Center (CXC).

Facilities: SDSS, CHANDRA (CIAO), GMRT, VLA, CXO.

Software: SPAM (Intema 2014), PROFFIT (Eckert et al. 2011).

\section{ORCID iDs}

M. B. Pandge (i) https://orcid.org/0000-0002-9699-6257 S. S. Sonkamble (1) https://orcid.org/0000-0001-8985-8596 Somak Raychaudhury (1) https://orcid.org/0000-00024864-4046

\section{References}

Bîrzan, L., McNamara, B. R., Nulsen, P. E. J., Carilli, C. L., \& Wise, M. W. 2008, ApJ, 686, 859

Bîrzan, L., Rafferty, D. A., McNamara, B. R., Wise, M. W., \& Nulsen, P. E. J. 2004, ApJ, 607, 800

Bîrzan, L., Rafferty, D. A., Nulsen, P. E. J., et al. 2012, MNRAS, 427, 3468 Blundell, K. M., Rawlings, S., \& Willott, C. J. 1999, AJ, 117, 677

Cavagnolo, K. W., McNamara, B. R., Nulsen, P. E. J., et al. 2010, ApJ, 720,1066

Chon, G., Böhringer, H., Krause, M., \& Trümper, J. 2012, A\&A, 545, L3

David, L. P., Jones, C., Forman, W., et al. 2009, ApJ, 705, 624

David, L. P., O'Sullivan, E., Jones, C., et al. 2011, ApJ, 728, 162

Dong, R., Rasmussen, J., \& Mulchaey, J. S. 2010, ApJ, 712, 883

Dunn, R. J. H., Allen, S. W., Taylor, G. B., et al. 2010, MNRAS, 404, 180

Dunn, R. J. H., \& Fabian, A. C. 2006, MNRAS, 373, 959

Eckert, D., Molendi, S., \& Paltani, S. 2011, A\&A, 526, A79

Eke, V. R., Frenk, C. S., Baugh, C. M., et al. 2004, MNRAS, 355, 769

Enßlin, T. A., \& Gopal-Krishna 2001, A\&A, 366, 26

Giacintucci, S., O'Sullivan, E., Clarke, T. E., et al. 2012, ApJ, 755, 172

Giacintucci, S., O'Sullivan, E., Vrtilek, J., et al. 2011, ApJ, 732, 95

Giacintucci, S., Vrtilek, J. M., Murgia, M., et al. 2008, ApJ, 682, 186

Gitti, M., O'Sullivan, E., Giacintucci, S., et al. 2010, ApJ, 714, 758

Hudson, D. S., \& Henriksen, M. J. 2003, ApJL, 595, L1

Hudson, D. S., Henriksen, M. J., \& Colafrancesco, S. 2003, ApJ, 583, 706

Intema, H. T. 2014, BASI, 13, 1

Intema, H. T., Jagannathan, P., Mooley, K. P., \& Frail, D. A. 2017, A\&A, 598, A78

Jetha, N. N., Hardcastle, M. J., Babul, A., et al. 2008, MNRAS, 384, 1344

Jones, C., Forman, W., Vikhlinin, A., et al. 2002, ApJL, 567, L115

Kempner, J. C., Blanton, E. L., Clarke, T. E., et al. 2004, in The Riddle of Cooling Flows in Galaxies and Clusters of Galaxies, ed. T. Reiprich, J. Kempner, \& N. Soker

Machacek, M., Nulsen, P. E. J., Jones, C., \& Forman, W. R. 2006, ApJ, 648, 947

McDonald, M., Veilleux, S., Rupke, D. S. N., \& Mushotzky, R. 2010, ApJ, 721,1262

McNamara, B. R., Wise, M., Nulsen, P. E. J., et al. 2000, ApJL, 534, L135

Mittal, R., Hudson, D. S., Reiprich, T. H., \& Clarke, T. 2009, A\&A, 501, 835

O'Sullivan, E., Giacintucci, S., David, L. P., et al. 2011, ApJ, 735, 11

O'Sullivan, E., Kolokythas, K., Kantharia, N. G., et al. 2018, MNRAS, 473,5248

O'Sullivan, E., Ponman, T. J., Kolokythas, K., et al. 2017, MNRAS, 472, 1482

Ogrean, G. A., Brüggen, M., van Weeren, R., et al. 2011, MNRAS, 414, 1175

Panagoulia, E. K., Fabian, A. C., \& Sanders, J. S. 2014, MNRAS, 438, 2341

Pandge, M. B., Vagshette, N. D., David, L. P., \& Patil, M. K. 2012, MNRAS, 421, 808

Pandge, M. B., Vagshette, N. D., Sonkamble, S. S., \& Patil, M. K. 2013, Ap\&SS, 345, 183

Perley, R. A., \& Butler, B. J. 2017, ApJS, 230, 7

Peterson, J. R., \& Fabian, A. C. 2006, PhR, 427, 1

Rafferty, D. A., McNamara, B. R., Nulsen, P. E. J., \& Wise, M. W. 2006, ApJ, 652,216

Rines, K. J., Geller, M. J., Diaferio, A., \& Hwang, H. S. 2016, ApJ, 819, 63

Sarazin, C. L. 1988, S\&T, 76, 639

Smith, R. J., Hudson, M. J., Nelan, J. E., et al. 2004, AJ, 128, 1558

Smith, R. K., Brickhouse, N. S., Liedahl, D. A., \& Raymond, J. C. 2001, ApJL, 556, L91

Sun, M., Voit, G. M., Donahue, M., et al. 2009, ApJ, 693, 1142

Trinchieri, G., Breitschwerdt, D., Pietsch, W., Sulentic, J., \& Wolter, A. 2007, A\&A, 463, 153

Trinchieri, G., \& Pietsch, W. 2000, A\&A, 353, 487

Vaddi, S., O’Dea, C. P., Baum, S. A., et al. 2016, ApJ, 818, 182

Vagshette, N. D., Naik, S., Patil, M. K., \& Sonkamble, S. S. 2017, MNRAS, 466, 2054

Vagshette, N. D., Sonkamble, S. S., Naik, S., \& Patil, M. K. 2016, MNRAS, 461,1885

van Weeren, R. J., Röttgering, H. J. A., Intema, H. T., et al. 2012, A\&A, 546, A124

Wegner, G., Colless, M., Saglia, R. P., et al. 1999, MNRAS, 305, 259

Young, A., Rudnick, L., Katz, D., et al. 2005, ApJ, 626, 748 